\title{
Adaptation to Visuomotor Rotations Remaps Movement Vectors, Not Final Positions
}

\author{
Jinsung Wang and Robert L. Sainburg \\ Department of Kinesiology, The Pennsylvania State University, University Park, Pennsylvania 16802
}

\begin{abstract}
When exposed to novel visuomotor rotations, subjects readily adapt reaching movements, such that the virtual display of the hand is brought to the target. Whereas this clearly reflects remapping of the relationship between hand movements and the visual display, the nature of this remapping is not well understood. We now examine whether such adaptation results in remapping of the position of the visually displayed target and the final limb position or between the target vector and the movement vector. The latter is defined relative to a starting position, whereas the former should be independent of the starting position. Subjects first adapted to a $30^{\circ}$ rotation during reaching movements made from a single starting location to four different target locations. After adaptation, generalization trials were introduced, during which reaching movements were made under the same visual rotation condition but started from one of two locations outside the practiced workspace. These trials were directed to either the previously practiced targets or new targets that reflected the direction and distance of the practiced trials. Generalization was greatest for movements made in similar directions, regardless of changes in spatial location. Most significantly, when reaching to the previously adapted targets, subjects did not reach to the previously learned limb positions but rather to positions that reflected a near $30^{\circ}$ rotation of the new target vector. These results indicate that learned visuomotor rotations remap the representations of movement vectors and not final positions of the limb in the workspace.
\end{abstract}

Key words: human; motor learning; motor control; generalization; reaching; movement representation

\section{Introduction}

Previous studies of motor learning have addressed how individuals adapt to novel visuomotor transformations, imposed by rotating a virtual display of limb position (Pine et al., 1996; Ghahramani and Wolpert, 1997; Krakauer et al., 1999; Baraduc and Wolpert, 2002; Sainburg, 2002; Sainburg and Wang, 2002; Tong et al., 2002; Wang and Sainburg, 2003, 2004). However, the mechanisms that underlie the associated remapping of the relationship between hand movements and the visual display remain uncertain. A reasonable assumption is that a given location in display space is mapped to a new location in motor space. For example, the position of the displayed target becomes associated with a new limb configuration. This idea is consistent with theories of motor control that posit that movements are represented as intended final limb postures, such as certain versions of the equilibrium point hypothesis that imply endpoint control (Feldman, 1966; Polit and Bizzi, 1978, 1979; Latash and Gottleib, 1990; Jaric et al., 1992, 1994) and Rosenbaum's posture-based model of motion planning (Rosenbaum et al., 1993, 1999; Meulenbroek et al., 2001). It is also possible that the movement vector is remapped relative to target vectors. This idea is consistent with the vectorial hypothesis of movement, which proposes that tar-

Received Dec. 8, 2004; revised March 10, 2005; accepted March 15, 2005.

This research was supported by National Institutes of Health Grant R01HD39311 and National Research Service Award 1-F32-NS-46239-1.

Correspondence should be addressed to Dr. Jinsung Wang, Department of Kinesiology, 266 Recreation Building, Pennsylvania State University, University Park, PA 16802. E-mail: jinsung@psu.edu. D0I:10.1523/JNEUROSCI.5000-04.2005

Copyright $\odot 2005$ Society for Neuroscience $\quad$ 0270-6474/05/254024-07\$15.00/0 geted movements are represented as intended direction and distance from a given starting location (Gordon et al., 1994a,b; Vindras et al., 1998; Messier and Kalaska, 1999; Krakauer et al., 2000; Sainburg et al., 2003). These ideas lead to different predictions about how adaptation to visuomotor rotations will generalize when subjects are required to move to previously adapted targets from new start positions. According to the vectorial hypothesis, learned rotations should be preserved for movements made in similar directions, such that subjects rotate movement directions in accord with the adapted visuomotor rotation. Alternatively, the position-remapping model predicts that subjects will move to the previously learned final positions, regardless of changes in start position.

We now directly test these hypotheses. After adaptation to a $30^{\circ}$ visuomotor rotation, subjects continued to make movements toward the practiced target, although occasionally, a trial began from a start location outside the practiced space to either one of the previously practiced targets or a new target reflecting the practiced direction and distance. If subjects learned the rotation by remapping limb position in relation to target position, movements toward the practiced targets should end at or near the previously learned final positions. In addition, movements toward targets distant from the practiced space should show less generalization than to those closer to the practiced space. In contrast, if subjects remapped target direction and distance to movement direction and distance, then the greatest generalization should occur to movements with the same direction and distance, although they are located in more distant regions of space. Movements directed toward the previously practiced targets should also reflect generalization of direction, which by design 


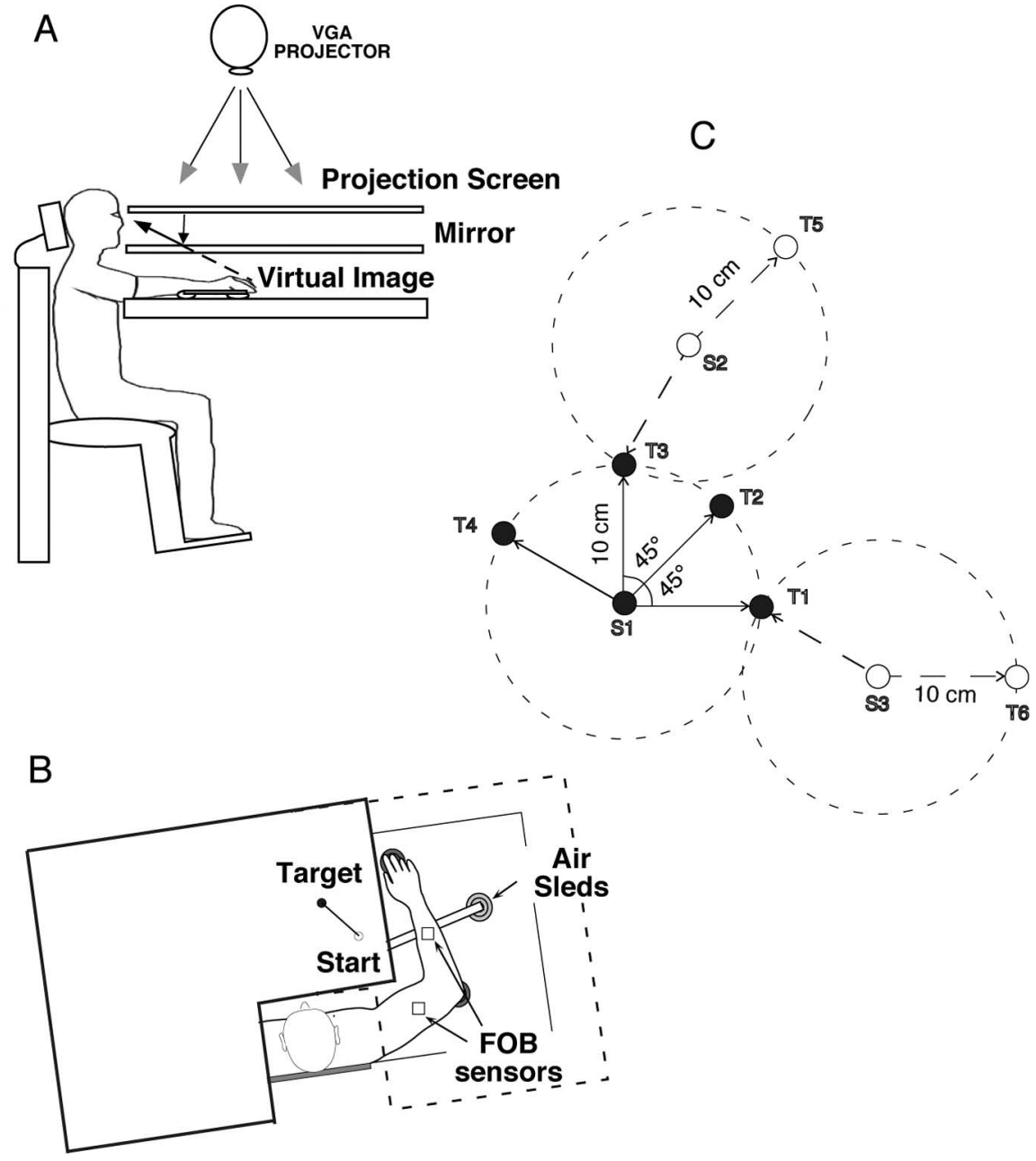

Figure 1. A, Side view. Subjects were seated in a dentist-type chair with the arm supported by an air-jet system that removed the effects of friction on arm movement. Targets and the cursor representing hand position were back-projected on a screen placed above the arm. A mirror placed below this screen reflected the image, such that the projection was perceived in the plane of the arm. $\boldsymbol{B}$, Top view. The positions of the Flock of Birds (FOB) sensors are shown. $\boldsymbol{C}$, Target schemes. Subjects first adapted to $30^{\circ}$ $\mathrm{CCW}$ rotation by making movements from $\mathrm{S} 1$ to training targets $\mathrm{T1}$-T4. Generalization trial movements were made from two new start locations, S2 and S3, to two practiced target locations, T1 and T3, or to two new target locations, T5 and T6.

ment recording system. The position of the following three bony landmarks was digitized: (1) index fingertip, (2) the lateral epicondyle of the humerus, and (3) the acromion, directly posterior to the acromio-clavicular joint. As sensor data were received from the Flock of Birds, the position of these landmarks was computed by our custom software. For detailed information, see Sainburg and Wang (2002).

\section{Experimental design}

The experiment consisted of baseline (no visual rotation) and adaptation (visual rotation) sessions (40 and 280 trials, respectively). During the adaptation session, the position of the cursor was rotated $30^{\circ}$ counterclockwise (CCW) relative to the start circle. For each trial, one of four training targets (Fig. 1C, T1-T4, $2 \mathrm{~cm}$ in diameter), presented in a pseudorandom sequence, was displayed before movement. Subjects were instructed to move straight from the starting circle (S1) to the target using a single, rapid motion in response to an auditory "go" signal.

The design of this task is based on the idea that the pattern of generalization exhibited after learning can shed light on how that learning is represented in the CNS. In this experiment, we tested two alternative hypotheses for how visuomotor rotations are represented: (1) positional hypothesis, in which subjects remap final limb positions in relation to the visually displayed targets and (2) vectorial hypothesis, in which subjects remap the direction and distance of the movement vector in relation to the direction and distance of the target vector. The latter is differentiated by the role of initial position information and by an independence of location in the workspace. A target vector is defined as a straight movement made from the starting circle to the target position in visual space, whereas a movement vector is defined as that made from the starting circle to the actual final hand position.

\section{Adaptation trials}

would direct the movement away from the previously experienced final position.

\section{Materials and Methods \\ Subjects}

Subjects were seven neurologically intact right-handed adults (four female, three male), aged 18-30 years old. Subjects were recruited from the university community and were paid for their participation. Informed consent was solicited before participation. Right handedness was assessed using the 10-item version of the Edinburgh inventory (Oldfield, 1971).

\section{Apparatus}

Subjects sat facing a table with the right arm supported over a horizontal surface, positioned just below shoulder height, by a frictionless air-jet system (Fig. 1A). A start circle, target, and cursor representing the index hand position were projected on a horizontal back-projection screen positioned above the arm (Fig. $1 \mathrm{~B}$ ). A mirror, positioned parallel and below this screen, reflected the visual display so as to give the illusion that the display was in the same horizontal plane as the hand. Calibration of the display ensured that this projection was veridical. Position and orientation of each limb segment were sampled at $103 \mathrm{~Hz}$ using the Flock of Birds (Ascension-Technology, Burlington, VT) magnetic 6-DOF move-
During each movement, visual feedback was provided as a screen cursor. After the completion of each trial, feedback continued to be provided, allowing subjects to reposition the cursor within the start circle. During the adaptation session, all feedback during and between movements was rotated relative to the hand position. At the end of each trial, knowledge of results was provided in the form of a cursor path between the starting circle and the target and by points awarded for spatial accuracy (twodimensional distance between the target and the final hand position): 1 point for accuracy $<4 \mathrm{~cm}, 3$ points for accuracy $<2 \mathrm{~cm}$, and 10 points for accuracy $<1 \mathrm{~cm}$. No points were given for movements that took longer than $400 \mathrm{~ms}$.

\section{Generalization trials}

After adaptation to four targets in central workspace (120 total trials), we presented "generalization" trials, which were occasionally and randomly dispersed within a continued block of trials in the adapted workspace (Fig. 1C, S2, S3). These trials began from one of two start positions outside of and lateral to the originally practiced space. From this new start location (S1 or S2), either one of the previously practiced targets (T1and T3) or one of two new targets (T5 and T6) was presented. The purpose of using generalization trials was to examine how subjects planned movements when starting from new locations. For these trials, no visual (cursor) feedback was provided during the movement to dis- 
courage on-line corrections. After the completion of each trial, veridical feedback (i.e., no visual rotation) was provided to the subjects to guide return of the cursor to the start circle. Whereas the cursor was visible within the start circle, cursor feedback was removed at movement onset. The starting positions for generalization trials are shown in Figure 1C. Subjects were completely aware of the changes in start position for these trials.

The location of new starting circles $(\mathrm{S} 2, \mathrm{~S} 3)$ was specifically determined to test our two alternative hypotheses regarding how visuomotor rotations are represented in the CNS. Our predictions are based on the idea that generalization should be greatest for movements that change the least in the parameter space that is modified during the learning process (Imamizu et al., 1998). Two targets (T5 and T6) were most distant in terms of workspace location but shared the same direction and distance relative to the new start position of the practiced targets.

In contrast, the previously experienced targets (T1 and T3) could be associated with either the limb configuration that was previously associated with those targets or a new final limb position associated with the learned directional rotation. Our predictions, based on the two hypothesized learning schemes, are as follows.

Positional representation. This idea suggests that subjects learn to associate a limb configuration (final position) with the rotated visual display. If this is the case, we predict that, when moving toward the previously learned targets from the new start positions, subjects should direct their hands toward the previously learned final posture (Fig. $2 \mathrm{~B}$ ). In addition, when reaching toward the new, more distant targets (T5 and T6), generalization in position should degrade because of the large change in position, and thus, movements should be directed near the veridical position of the target. However, we cannot speculate on the exact pattern of transfer expected under this condition.

Vectorial representation. This idea suggests that subjects learn to associate a target direction and distance with a movement direction and distance. For example, movements toward target 1 should show substantial generalization (reflected by the $30^{\circ}$ rotation) because these targets share the direction and distance of the learned targets. Movements directed toward target $\mathrm{T} 3$, however, reflect a new direction and should thus show less directional transfer. Nevertheless, if subjects learn a directional rotation rather than a positional mapping, movements toward this target should be rotated away from the previously learned final position (Fig. $2 C$ ). For movements made toward targets 5 and 6 , we expect extensive generalization because they share the directions and distances of previously practiced targets, although these targets are farthest away in the workspace.

In this way, we are able to clearly differentiate how subjects represent the visuomotor rotation. For all targets, we have opposite predictions for each representation scheme. The close targets can be associated with the exact same limb configurations (positions) as those experienced during training, and these positions reflect an opposite rotation relative to the new start position. The far targets can be associated with the exact same direction and distance as the trained targets, but these movements would be associated with a large difference in limb configuration and workspace location. Thus, the pattern of transfer observed in this study should clearly differentiate whether subjects remap the positions in the workspace relative to the visual display or the directional rotation relative to a given start position, regardless of directional changes.

\section{Data analysis}

Two measures of performance were calculated: hand-path direction error at peak tangential arm velocity $\left(V_{\max }\right)$ and final position error. Direction error was calculated as the angular difference between the vectors defined by the target and by the hand-path position at movement start and at $V_{\max }$. Final position error was calculated as the two-dimensional distance between the index hand at movement termination and the center of the target. To assess the amount of generalization from reaching movements made toward the four training targets to those made toward the four generalization targets, each subject's performance was quantified in the following two steps. First, the level of performance after adaptation to the visual rotation was quantified as the mean direction error and the mean final position error measure for the last 35 trials of reaching movements made toward each training target (i.e., all of the trials after the first 120 trials). Second, to assess the difference between this adapted performance and the performance during generalization trials, mean adapted performance measures for each training target, calculated in step 1 , were subtracted from the measures of generalization trials for its corresponding target to yield "difference between adapted performance and generalization trial performance" values (for movements from S2 to T3, the overall mean values across all training targets were subtracted). Then, for each subject, a single mean value for the five generalization trials for each target direction was calculated for each task performance measure. A repeated-measures ANOVA was conducted to examine the main effect of generalization targets. Tukey's post hoc tests were used to assess the differences among the four generalization targets.

\section{Results}

\section{Visuomotor adaptation to training targets}

Figure 3 shows typical hand paths of one representative subject during the initial and final phases of the adaptation session. As expected, these paths are initially directed CCW to the target (left column), with a large "hook" at the end of motion that reflects an error correction. After adaptation to the visual rotation, these movements become relatively straight and substantially more accurate (right column). The mean \pm SE values of direction errors and final position errors, averaged across every five consecutive trials for each training target, are shown in Figure 4. Substantial improvement in both performance measures is observed by block 7 , after which the performance measures remain relatively stable until the end of the session. These findings show that subjects readily adapted to the imposed visuomotor rotation.

\section{Generalization of visuomotor adaptation to previously practiced targets}

In this experiment, all subjects first performed 120 trials of reaching movement to the four training targets from the initial start location (S1). After this, generalization trials for the two previously experienced targets (five trials per target) were presented randomly, such that the subject reached from a new start location (S2 or S3) to either T1 or T3. It should be stressed that, during 


\section{TRAINED TARGETS}

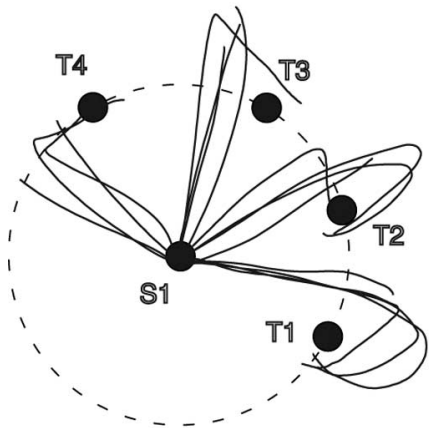

\section{Initial Exposure}

\section{Adapted}

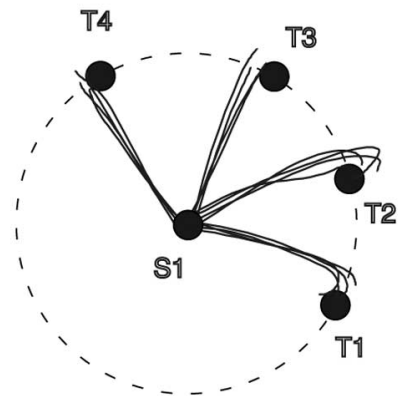

Figure 3. Hand paths of a representative subject during the adaptation session (from trial 1 to trial 120). On the initial exposure, hand paths are initially directed $\mathrm{CCW}$ to the target, with a large "hook" at the end of motion that reflects an error correction (left). After adaptation to the visual rotation, hand paths become relatively straight and substantially more accurate (right).
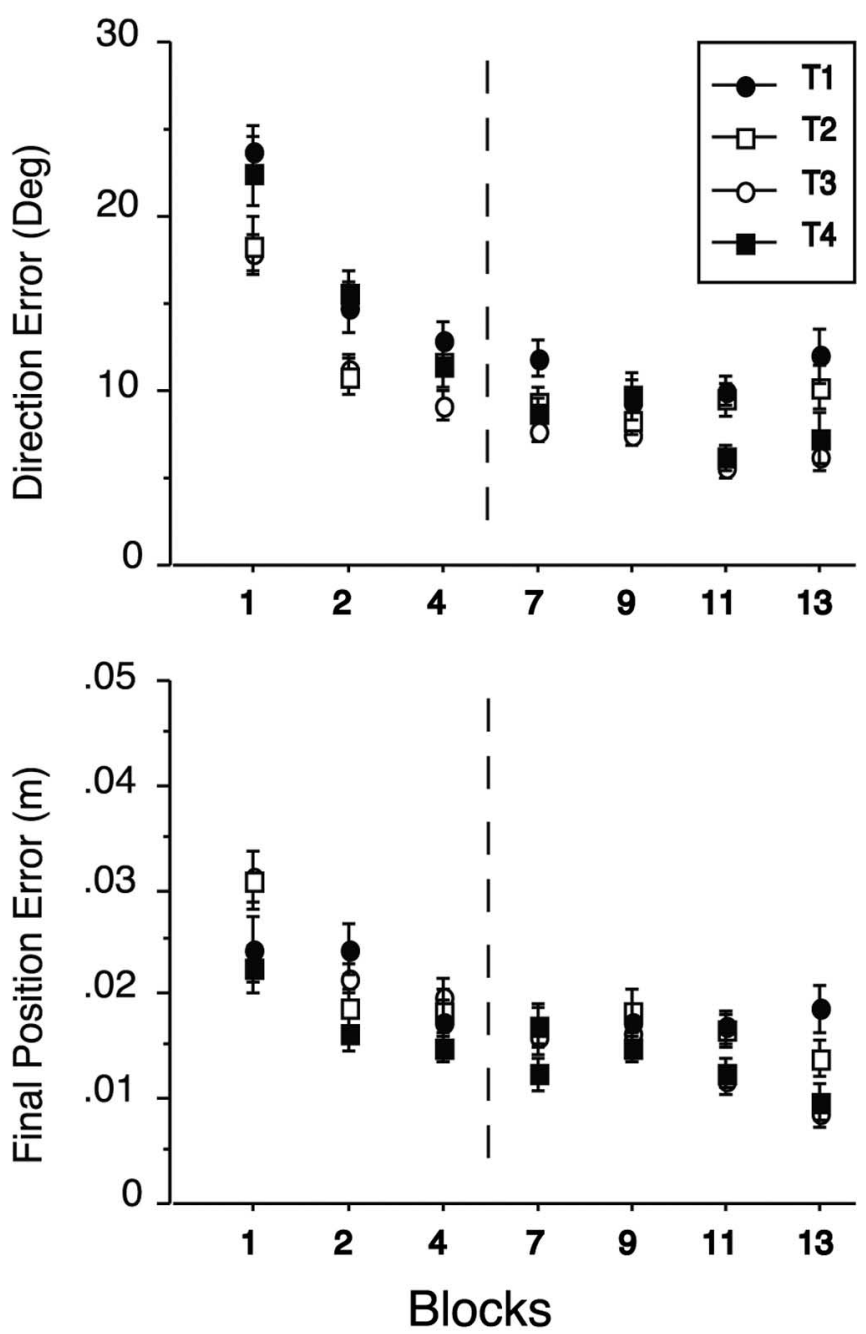

Figure 4. Mean performance measures of direction error at $V_{\max }$ and final position error. Every data point in each block represents the mean of five consecutive trials averaged across all subjects (mean $\pm \mathrm{SE}$ ). Substantial improvement in both performance measures is observed by block 7, after which the performance measures remain relatively stable until the end of the session. Vertical broken line indicates the point after which generalization trials were introduced.

\section{Generalization Trials}

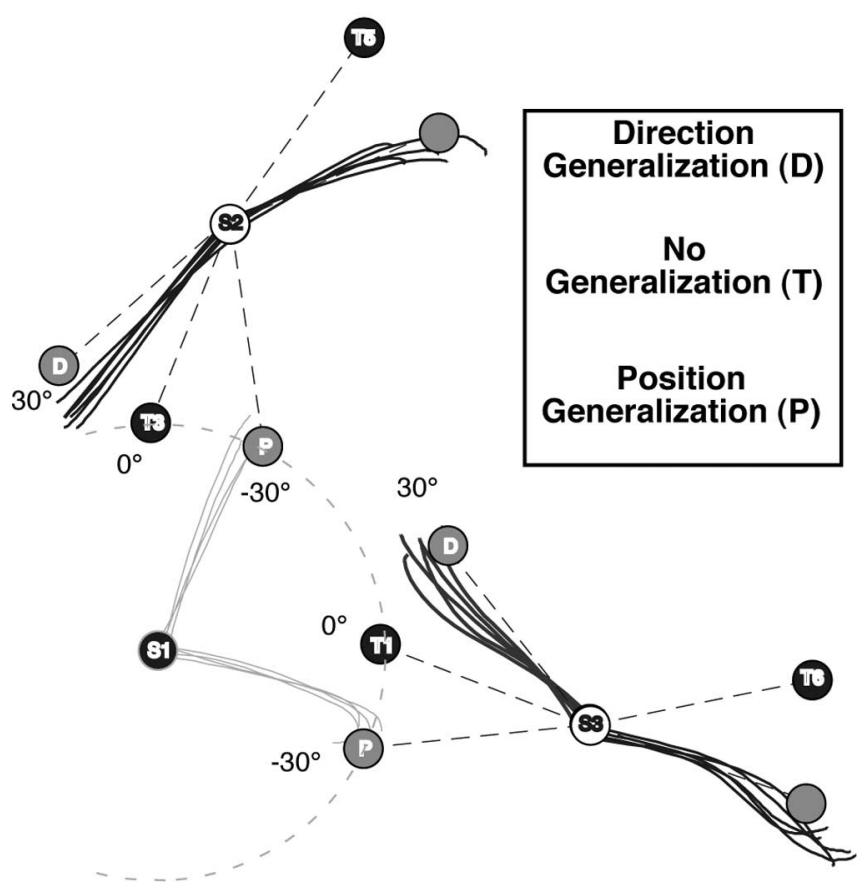

Figure 5. Hand paths of a representative subject during the generalization trial performance. Black lines represent the hand paths during generalization trials to practiced targets, whereas gray lines represent sample hand paths of adapted performance. Substantial generalization toward the practiced target directions, and not toward the practiced final positions, is clearly observed in these hand paths.

these generalization trials, neither feedback nor knowledge of results was provided. These targets were the same as targets 1 and 3 , which were adapted during adaptation trials made from start position S1. In Figure 5, these targets are shown, along with sample hand paths of adapted performance, shown in gray. In this figure, the two alternative predictions for hand movements are shown as grayed "targets." Thus, the gray targets marked as " $\mathrm{D}$ " represent the predictions of the direction generalization hypothesis, whereas the ones marked with "P" represent the position generalization hypothesis. If no generalization occurred, subjects would be expected to direct hand movements toward the visually presented target (T1 or T3).

Typical results are indicated by the hand paths starting from locations S2 and S3. Rather than being directed toward the final hand positions previously associated with these targets, movements were made in the opposite direction, reflecting generalization of the $30^{\circ}$ clockwise $(\mathrm{CW})$ rotation. Most notable was the fact that movements from start position 3 toward target 1 reflected a previously practiced direction (from start position 1 to target 4) and showed nearly complete directional generalization. Movements made from start position 2 to target 3, in contrast, reflected an unpracticed direction. Hand movements, in this case, showed incomplete directional generalization $\left(\sim 20^{\circ}\right)$ but clearly were directed away from the previously adapted final position associated with that target. Our results, therefore, reveal unequivocal generalization of visuomotor rotations in terms of movement direction but not in terms of final position.

\section{Generalization of visuomotor adaptation to} unpracticed targets

After the first 120 trials, generalization trials for the two unpracticed targets (five trials per target) were also presented occasion- 

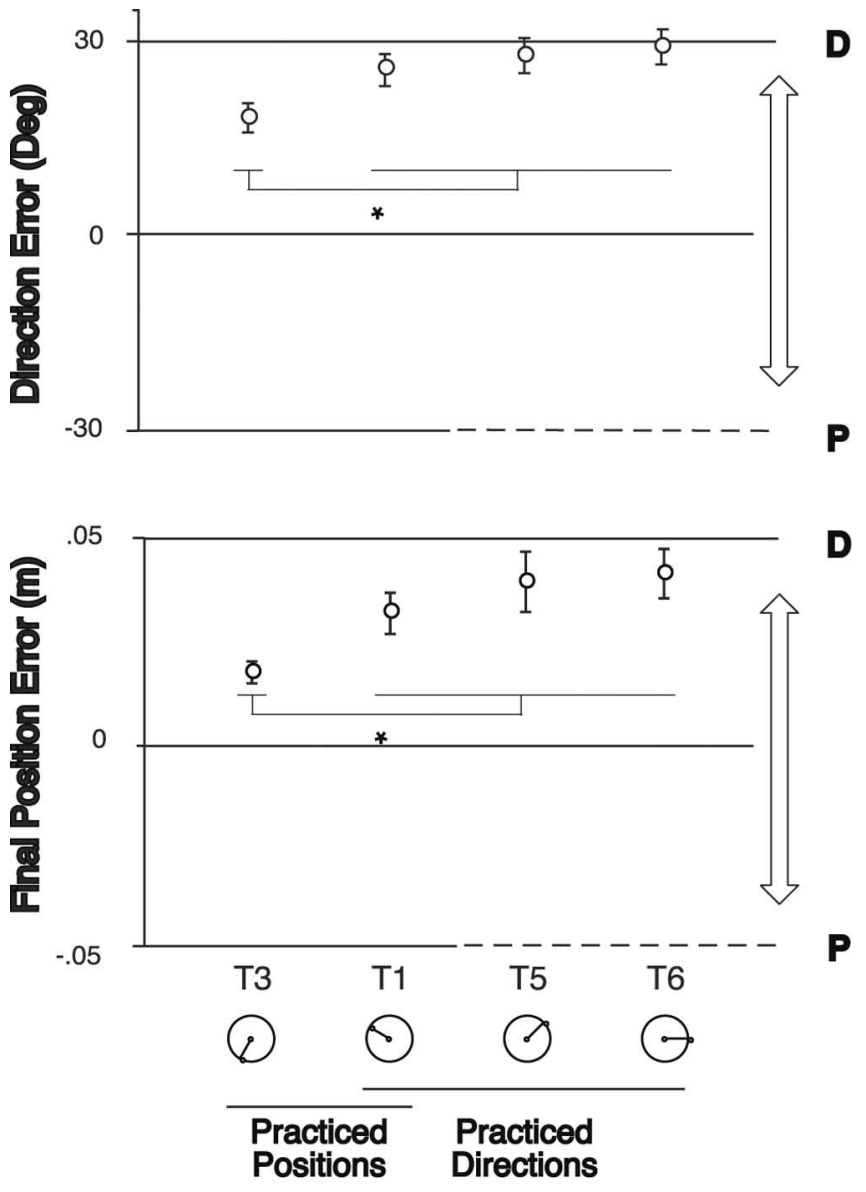

Figure 6. Direction errors and final position errors (mean $\pm 95 \%$ confidence interval) for each generalization target. The maximum and minimum on the $y$-axis represents the predicted errors for perfect generalization according to direction generalization (D) or position generalization (P) hypothesis, respectively. Confidence intervals of $95 \%$ indicate that the direction errors for $\mathrm{T} 5$ and $\mathrm{T} 6$ were not significantly different from $30^{\circ}$. ${ }^{*} p<0.05$ indicates a significant difference.

ally and randomly, such that the subject reached from a new start location (S2 or S3) to a new target location placed outside the previously experienced space (T5 or T6). These targets directed away from the adapted workspace and reflected previously practiced directions and distances. Illustrated in Figure 5, these generalization trial movements showed complete generalization, as indicated by fairly straight movements toward the $30^{\circ}$ rotation position. These data indicate substantial generalization of visuomotor rotations to movements that occur outside of the experienced workspace, when the movement direction and distance of the new target, relative to the new starting location, reflect the previously practiced direction and distance. They are in agreement with the vectorial hypothesis because, according to this hypothesis, the largest generalization should occur for movements made in directions and distances similar to those that have been experienced previously. These findings contrast with the position-remapping hypothesis, which predicts degraded generalization for movements made toward the new, more distant targets.

\section{Mean performance measures}

These findings were robust for all subjects, as revealed by Figure 6 , which shows the average (across subjects) direction errors and final position errors [mean $\pm 95 \%$ confidence interval (CI)] for each generalization target. We plotted direction errors and final position errors relative to the absolute location of the visually presented target. The zero error line (middle of both plots) reflects the prediction for no generalization conditions. The maximum and minimum on the $y$-axis represents the predicted errors for perfect generalization according to direction or final position hypothesis, respectively (i.e., $\pm 30^{\circ}$ for direction errors, $\pm 0.05 \mathrm{~m}$ for final position errors). Repeated-measures ANOVA revealed a significant main effect of these generalization targets $(p<0.05)$, and the Tukey's post hoc tests indicated that, for both performance measures, target 3 was significantly different from the other three targets $(p<0.05)$, which were not significantly different from each other. It was also revealed, using the 95\% CIs, that the direction errors for T5 and T6 were not significantly different from $30^{\circ}$ (mean \pm CI, $27.8 \pm 2.5$ and $28.8 \pm 2.7$, respectively), whereas the direction errors for T1 and T3 and the final position errors for all targets were significantly different from $30^{\circ}$ or $0.05 \mathrm{~m}$, respectively.

These results indicate that learning generalized in terms of movement direction and not final position. Generalization in direction was, not surprisingly, greatest for those movements made in the same direction as previously trained targets, although those targets were farthest away from the adapted workspace (targets 5 and 6). Directional generalization was least for target 3 , which was toward an unpracticed direction.

\section{Discussion}

In this study, we investigated whether adaptation to a visuomotor rotation produces a remapping between the target position in visual space and the final hand position or between the target vectors and the hand-movement vectors. We predicted that, if visuomotor adaptation produces a remapping between the target and final hand positions, reaching movements from a new starting location to a previously practiced target location should be made toward the previously adapted final hand position. If, however, visuomotor adaptation produces a remapping between the target and movement vectors, those reaching movements should be made toward a new final location that matches the previously practiced direction (i.e., a movement vector rotated $30^{\circ} \mathrm{CW}$ relative to the target vector). In addition, the largest generalization should occur to a novel environment in which reaching movements are made from a new starting location to a new target location that matches the direction and distance of a previously practiced target. Our data demonstrated extensive generalization to the target locations that matched the practiced direction and not to the practiced final hand positions. These findings indicate unequivocal generalization of visuomotor rotations in terms of movement direction and indicate that such adaptation produces a remapping between target vectors and intended movement vectors.

Our finding that directional generalization was least for target 3 , an unpracticed direction, agrees with the findings of Krakauer et al. (2000), demonstrating that adaptation with a limited number of targets results in incomplete generalization to untrained directions. Adaptation to eight targets, in that study, resulted in $100 \%$ transfer to targets that deviated as much as $22.5^{\circ}$ from the trained directions. However, training to only four targets resulted in transfer of only $85 \%$ to targets that deviated by $22.5^{\circ}$ and only $65 \%$ for targets that deviated by $45^{\circ}$ from the trained directions. Our data indicate transfer of $60 \%$ generalization to a direction that deviated nearly $90^{\circ}$ from its closest training direction (S1 to T4). This finding extends that of Krakauer et al. by showing that limited training experience can lead to substantial generalization 
across $90^{\circ}$, even when the transfer movement is performed in a different region of the workspace. In addition, our findings indicate $100 \%$ transfer for movements made in the same direction of training but that are positioned in an untrained region of the workspace. This result appears to contradict the recent findings by Baraduc and Wolpert (2002) that the extent of direction generalization sharply diminishes with changes in initial arm configurations. However, that study quantified the aftereffects of transfer when exposed in the new region of space to the null condition. In contrast, we examined the degree to which learning is preserved when exposed to the previously experienced visuomotor transform during reaching movement made in a new region of space. These two measures clearly do not represent the same processes, because we have shown previously that performance can transfer without the occurrence of aftereffects (Sainburg and Wang, 2002; Wang and Sainburg, 2004). Therefore, our findings support and extend those of Krakauer et al. (2000) and Baraduc and Wolpert (2002) by demonstrating substantial transfer of visuomotor transformations across both movement directions and workspace location.

\section{Vectorial planning versus positional planning}

Our results provide strong support to the vectorial hypothesis of movement planning (Gordon et al., 1994a,b; Vindras et al., 1998; Messier and Kalaska, 1999; Krakauer et al., 2000). According to this hypothesis, the control system combines information regarding the location of the target and the initial position of the hand to form a hand-centered plan of the intended movement trajectory as a direction and extent in extrinsic space. We believe that such plans are formed during visuomotor adaptation, and they can be applied to novel conditions to facilitate reaching movements made from different hand positions by adjusting required dynamic strategies in accord with changes in limb configuration. Although our current findings do not directly address the origin of the learned coordinate transform, previous findings from our laboratory suggest that such visuomotor maps have an origin at the starting finger location. In a recent study, we altered initial position information by changing the relationship of the hand position to the cursor location before occasional "catch" trials (Sainburg et al., 2003). The position of the hand was systematically shifted relative to the baseline start position (i.e., shifted $3.5 \mathrm{~cm}$ anterior, posterior, left, or right to the baseline position), but the cursor location was always located within the baseline start circle. We examined whether subjects moved toward the same final position, regardless of changes in hand start location, or whether subjects made the same displacement vector, regardless of errors in final position. Our results indicated that subjects maintained the direction of hand motion regardless of errors in final position, thus supporting the vectorial planning hypothesis. Movements from hand locations that were orthogonally displaced relative to the target vector were made with the same direction and distance as that of baseline movements. Thus, subjects appeared to adjust the movement plan to the new location of the hand (vector origin). Interestingly, for hand positions that were displaced parallel to the target vector, movement distance varied with initial start position in accord with the finger position, reflecting the use of proprioceptive feedback to adjust final position. In fact, Ghez and colleagues have shown previously that late modifications can be used to "update" the ongoing movement to correct for errors in the initial movement plan (Gordon and Ghez, 1987; Krakauer et al., 2002). This has been most consistently demonstrated for adjusting movement extent during targeted tasks (Brown and Cooke, 1981, 1984, 1986; Gordon and Ghez, 1987).

An alternative to the idea that movements are planned as displacement vectors posits that movements are represented as intended final hand positions or as limb postures (Feldman, 1966; Polit and Bizzi, 1978, 1979; Latash and Gottleib, 1990; Jaric et al., 1992, 1994; Rosenbaum et al., 1993, 1999; Meulenbroek et al., 2001). For example, Jaric et al. (1992, 1994) provided evidence for such positional control through a study in which different groups of subjects learned to make single joint movements from different start locations to either (1) a fixed position in space or (2) a fixed distance from each start location. In this study, subjects adapted better to the fixed position task, thus the authors concluded that movements are represented as intended final limb positions. In contrast to this interpretation, our current findings indicate that learned visuomotor rotations remap the representations of movement vectors and not final positions of the limb in the workspace. These findings, thus, leave open the question of the role of final position in movement planning and representation mechanisms.

\section{Independent mechanisms for control of trajectory and posture}

The discrepancy between vectorial and positional control theories is best understood by considering evidence that both trajectory and final limb position may be independently represented in the CNS. The seminal studies by Lackner and DiZio (Lackner and DiZio, 1994; DiZio and Lackner, 1995) showed independence in final position and trajectory control. In these studies, subjects reached to a target without visual feedback during adaptation to coriolis force fields, applied by a slowly revolving room (Lackner and DiZio, 1994). On initial exposure, subjects' hand paths were curved and inaccurate. When subjects were able to touch the target at the end of motion, movements became progressively straighter and directed toward the target with practice. However, when such haptic information was not available, movements became straight but were not directed toward the target. Thus, adaptation of trajectory and final position were clearly mediated by independent processes. A later study of interlimb transfer corroborated these studies by showing that final position, but not trajectory, transferred to the nonexposed limb (DiZio and Lackner, 1995), a finding supported by a number of our recent findings (Sainburg and Wang, 2002; Wang and Sainburg, 2003, 2004). Substantial evidence suggests that control of limb trajectory and final position are not only separately represented within each limb but that these distinctions have become further consolidated through lateral specialization in humans (Sainburg and Kalakanis, 2000; Bagesteiro and Sainburg, 2003; Haaland et al., 2004; Sainburg and Schaefer, 2004).

Our current findings, indicating that learning of visuomotor rotations seems to code trajectory and not final position, suggest that such adaptations preferentially affect the trajectory control feature of the system. This could occur because such control is likely to occur upstream to the processes that use feedback to control final position. Indeed, the findings described above suggest that control of final position is dependent on feedbackmediated, closed-loop processes, whereas control of trajectory appears to occur primarily through open-loop processes that anticipate impending dynamic conditions (Sainburg et al., 1993, 1995, 1999). Our current findings provide strong evidence that adaptation to visuomotor rotations occurs specifically through adjusting the trajectory planning process. In addition, because final positions are not remapped during this process, our current 
findings provide additional support to the idea that trajectory and final position are represented and controlled through independent neural processes.

\section{References}

Bagesteiro LB, Sainburg RL (2003) Nondominant arm advantages in load compensation during rapid elbow joint movements. J Neurophysiol 90:1503-1513.

Baraduc P, Wolpert DM (2002) Adaptation to a visuomotor shift depends on the starting posture. J Neurosci 88:973-981.

Brown SH, Cooke JD (1981) Responses to force perturbations preceding voluntary human arm movements. Brain Res 220:350-355.

Brown SH, Cooke JD (1984) Initial agonist burst duration depends on movement amplitude. Exp Brain Res 55:523-527.

Brown SH, Cooke JD (1986) Initial agonist burst is modified by perturbations preceding movement. Brain Res 377:311-322.

DiZio P, Lackner JR (1995) Motor adaptation to Coriolis force perturbations of reaching movements: endpoint but not trajectory adaptation transfers to the nonexposed arm. J Neurophysiol 74:1787-1792.

Feldman AG (1966) Functional tuning of the nervous system with control of movement or maintenance of a steady posture. II. Controllable parameters of the muscles. Biophysics 11:565-578.

Ghahramani Z, Wolpert DM (1997) Modular decomposition in visuomotor learning. Nature 386:392-395.

Gordon J, Ghez C (1987) Trajectory control in targeted force impulses. III. Compensatory adjustments for initial errors. Exp Brain Res 67:253-269.

Gordon J, Ghilardi MF, Ghez C (1994a) Accuracy of planar reaching movements. I. Independence of direction and extent variability. Exp Brain Res 99:97-111.

Gordon J, Ghilardi MF, Cooper SE, Ghez C (1994b) Accuracy of planar reaching movements. II. Systematic extent errors resulting from inertial anisotropy. Exp Brain Res 99:112-130.

Haaland KY, Prestopnik JL, Knight RT, Lee RR (2004) Hemispheric asymmetries for kinematic and positional aspects of reaching. Brain 127:1145-1158.

Imamizu H, Uno Y, Kawato M (1998) Adaptive internal model of intrinsic kinematics involved in learning an aiming task. J Exp Psychol Hum Percept Perform 24:812-829.

Jaric S, Corcos DM, Latash ML (1992) Effects of practice on final position reproduction. Exp Brain Res 91:129-134.

Jaric S, Corcos DM, Gottlieb GL, Ilic DB, Latash ML (1994) The effects of practice on movement distance and final position reproduction: implications for the equilibrium-point control of movements. Exp Brain Res 100:353-359.

Krakauer JW, Ghilardi MF, Ghez C (1999) Independent learning of internal models for kinematic and dynamic control of reaching. Nat Neurosci 2:1026-1031.

Krakauer JW, Pine ZM, Ghilardi MF, Ghez C (2000) Learning of visuomotor transformations for vectorial planning of reaching trajectories. J Neurosci 20:8916-8924.

Krakauer JW, Gordon J, Veytsman M, Ghez C (2002) Contributions of planning and updating to accuracy of reaching movements in normals and stroke. Soc Neurosci Abstr 28:169.4.
Lackner JR, DiZio P (1994) Rapid adaptation to Coriolis force perturbations of arm trajectory. J Neurophysiol 72:299-313.

Latash M, Gottleib G (1990) Hypothesis on the equilibrium point and variability of amplitude, speed and time of single-joint movement (in Russian). Biofizika 35:870-874.

Messier J, Kalaska JF (1999) Comparison of variability of initial kinematics and endpoints of reaching movements. Exp Brain Res 125:139-152.

Meulenbroek RG, Rosenbaum DA, Marteniuk RG, Bertram CP (2001) Planning reaching and grasping movements: simulating reduced movement capabilities in spastic hemiparesis. Motor Control 5:136-150.

Oldfield RC (1971) The assessment and analysis of handedness: the Edinburgh Inventory. Neuropsychologia 9:97-113.

Pine ZM, Krakauer JW, Gordon J, Ghez C (1996) Learning of scaling factors and reference axes for reaching movements. NeuroReport 7:2357-2361.

Polit A, Bizzi E (1978) Processes controlling arm movements in monkeys. Science 201:1235-1237.

Polit A, Bizzi E (1979) Characteristics of motor programs underlying arm movements in monkeys. J Neurophysiol 42:183-194.

Rosenbaum DA, Engelbrecht SE, Bushe MM, Loukopoulos LD (1993) A model for reaching control. Acta Psychol (Amst) 82:237-250.

Rosenbaum DA, Meulenbroek RJ, Vaughan J (1999) Remembered positions: stored locations or stored postures? Exp Brain Res 124:503-512.

Sainburg RL (2002) Evidence for a dynamic-dominance hypothesis of handedness. Exp Brain Res 142:241-258.

Sainburg RL, Kalakanis D (2000) Differences in control of limb dynamics during dominant and nondominant arm reaching. J Neurophysiol 83:2661-2675.

Sainburg RL, Schaefer SY (2004) Interlimb differences in control of movement extent. J Neurophysiol 92:1374-1383.

Sainburg RL, Wang J (2002) Interlimb transfer of visuomotor rotations: independence of direction and final position information. Exp Brain Res 145:437-447.

Sainburg RL, Poizner H, Ghez C (1993) Loss of proprioception produces deficits in interjoint coordination. J Neurophysiol 70:2136-2147.

Sainburg RL, Ghilardi MF, Poizner H, Ghez C (1995) Control of limb dynamics in normal subjects and patients without proprioception. J Neurophysiol 73:820-835.

Sainburg RL, Ghez C, Kalakanis D (1999) Intersegmental dynamics are controlled by sequential anticipatory, error correction, and postural mechanisms. J Neurophysiol 81:1040-1056.

Sainburg RL, Lateiner JE, Latash ML, Bagesteiro LB (2003) Effects of altering initial position on movement direction and extent. J Neurophysiol 89:401-415.

Tong C, Wolpert DM, Flanagan JR (2002) Kinematics and dynamics are not represented independently in motor working memory: evidence from an interference study. J Neurosci 22:1108-1113.

Vindras P, Desmurget M, Prablanc C, Viviani P (1998) Pointing errors reflect biases in the perception of the initial hand position. J Neurophysiol 79:3290-3294.

Wang J, Sainburg RL (2003) Mechanisms underlying interlimb transfer of visuomotor rotations. Exp Brain Res 149:520-526.

Wang J, Sainburg RL (2004) Limitations in interlimb transfer of visuomotor rotations. Exp Brain Res 155:1-8. 